\title{
FLAT AND FP-INJECTIVITY
}

\author{
SAROJ JAIN 1
}

\begin{abstract}
One of the main results of this paper is the characterization of left FP-injective rings as rings over which every finitely presented right module is torsionless. The necessity of the torsionless condition (in fact reflexivity) previously has been observed for special classes of FP-injective rings (e.g., self-injective rings (Kato and McRae), right and left coherent and self FP-injective rings (Stenström)). Furthermore, if the ring is left coherent, it is equivalent to say that every finitely presented right module is reflexive. We investigate the condition (called IF) that every injective right module is flat and characterize this via FP-injectivity. IF-rings generalize regular rings and QF-rings, and under certain chain or homological conditions coincide with regular or QF-rings (e.g., $w g l \operatorname{dim} R \leqq 1 \Rightarrow$ regular, noetherian, or right perfect $\Rightarrow Q F$ ).
\end{abstract}

1. Introduction. It is well known in QF-rings (Quasi-Frobenius) that finitely generated modules are reflexive. Also it is easy to see that over a regular ring (in the sense of von Neumann) that every finitely presented module is reflexive. In this paper, we show that the property of every finitely presented module being torsionless is a characterizing property of left FP-injective rings. (Recall the definition \$2.) For left coherent rings, we can strengthen this to the statement that every finitely presented right $R$-module is reflexive. This concept generalizes injective modules and self-injective rings, in particular, QF-rings. We also consider another generalization of QF-rings, namely right IF-rings. A ring $R$ is said to be right IF if every injective right $R$-module is flat. A theorem of Faith and Walker [5, Theorem 5.3] characterizes QF-rings with the property that every injective module is projective. Since any regular ring is right IF, the class of all right IF-rings is quite large. We connect right IF-rings with left FP-injectivity as follows: A left coherent ring is right IF iff $R$ is left

\section{Received by the editors January 29, 1973.}

AMS (MOS) subject classifications (1970). Primary 16A30, 16A50, 16A52; Secondary 16A34, 16A62.

Key words and phrases. QF-ring, IF-ring, self-injective ring, FP-injective ring, coherent ring, perfect ring, regular ring, semiprime Goldie ring, right and left annulets, injective module, FP-injective module, flat module, projective module, torsionless module, reflexive module, finitely presented module.

1 This paper is part of the author's doctoral dissertation written under the direction of Professor Carl Faith at Rutgers University, 1973. 
FP-injective. (Moreover left coherent is necessary for right self-injective right IF-rings.) We also characterize right IF-rings in a number of special cases including when $R$ is right perfect (in this case $R$ is QF) and $w g l R \leqq 1$ ( $R$ is regular in this case).

The author wishes to express her appreciation to her advisor, Professor Carl Faith, for his generous advice and encouragement for the preparation of this paper.

2. FP-injective and reflexive modules. $A$ left $R$-module is called left $F P$-injective iff $\operatorname{Ext}^{1}(F, M)=0$ for every finitely presented module $F$. A left $F P$-injective ring $R$ is left $F P$-injective as left $R$-module.

The following is clear (e.g. Gupta [8]).

Lemma 2.1. Let $\left\{M_{i}\right\}_{i \in I}$ be a family of left $R$-modules, then direct product $\prod M_{i}$ (respectively direct sum $\oplus M_{i}$ ) is left FP-injective iff each $M_{i}$ is left FP-injective.

We also use the following result which can be found in Auslander [1] and especially Lipman [10].

LEMMA 2.2. Let $R$ be any ring, and let

$$
F_{0} \rightarrow F_{1} \rightarrow M \rightarrow 0
$$

be an exact sequence of $R$-modules, where $F_{0}$ and $F_{1}$ are projective and of finite type. Let $N$ be the cokernel of the dual map $F_{1}^{*} \rightarrow F_{0}^{*}$. Then there is an exact sequence

$$
0 \rightarrow \operatorname{Ext}^{1}(N, R) \rightarrow M \rightarrow M^{* *} \rightarrow \operatorname{Ext}^{2}(N, R) \rightarrow 0 .
$$

In the next theorem, we refer to torsionless and reflexive modules in the sense that the canonical homomorphism $M \rightarrow M^{* *}$ is monomorphism and isomorphism respectively, where $M^{*}=\operatorname{Hom}_{R}(M, R)$.

THEOREM 2.3. $R$ is left FP-injective iff every finitely presented right $R$ module is torsionless.

Proof. Let $M$ be a finitely presented right $R$-module. Then by the above lemma, we have an exact sequence

$$
0 \rightarrow \operatorname{Ext}^{1}(N, R) \rightarrow M \rightarrow M^{* *} \rightarrow \operatorname{Ext}^{2}(N, R) \rightarrow 0 .
$$

$N$ being a cokernel of $F_{1}^{*} \rightarrow F_{0}^{*}$ is finitely presented. Thus $R$ left FPinjective implies $\operatorname{Ext}^{1}(N, R)=0$.

Thus $M \rightarrow M^{* *}$ is a monomorphism. Conversely, let every finitely presented right module be torsionless. Assuming ${ }_{R} M$ to be a finitely presented module, we have an exact sequence

$$
F_{0} \rightarrow F_{1} \rightarrow M \rightarrow 0 \text {, }
$$


where $F_{0}$ and $F_{1}$ are finitely generated free right $R$-modules. Then applying the dual functor $\operatorname{Hom}_{R}(, R)$ we get an exact sequence

$$
0 \rightarrow M^{*} \rightarrow F_{1}^{*} \rightarrow F_{0}^{*} \rightarrow N \rightarrow 0
$$

where $N=$ cokernel of $F_{1}^{*} \rightarrow F_{0}^{*}$.

Now applying the above lemma to the right half of the sequence (1), we get

$$
0 \rightarrow \operatorname{Ext}^{1}(M, R) \rightarrow N \rightarrow N^{* *},
$$

$M=$ cokernel of $F_{0}^{* *} \rightarrow F_{1}^{* *}$; now $N$ torsionless $\Rightarrow \operatorname{Ext}^{1}(M, R)=0$. Thus $R$ is left FP-injective.

COROLlary 2.4. A left coherent ring is left FP-injective iff every finitely presented right $R$-module is reflexive.

Proof. $\Leftarrow$ by Theorem 2.3.

$\Rightarrow \operatorname{Ext}^{1}(F, R)=0$ for every finitely presented left $R$-module $F$. Since $R$ is left coherent, this implies $\operatorname{Ext}^{n}(F, R)=0$ for every $n \geqq 1$. The result follows from the proof of Theorem 2.3.

COROLlary 2.5. In a left FP-injective ring, every finitely generated right ideal $I$ is right annulet.

The proof follows by the fact that $R / I$ is torsionless. The referee has remarked that the following proposition is also a consequence of Utumi's theorem [13], which implies that a left self-injective ring with zero singular ideal is regular.

Proposition 2.6. A left self-injective ring with no nil elements is regular.

Proof. By Corollary 2.5, $r l(I)=I$ for every finitely generated right ideal $I$ of $R$. Let $L=I \oplus l(I)$. Since $R$ has no nil elements, then $l(I)$ and hence $L$ is a two-sided ideal of $R$. Define $\sigma: I \oplus l(I) \rightarrow R$ by $a+b \rightarrow a, a \in I$, $b \in l(I)$. Then $\sigma$ is given by a right multiplication of an element $r$. Further $r$ is an idempotent and $I$ is generated by $r$.

COROLlary 2.7. A prime left self-injective ring $R$ is a field iff $R$ has no nil elements.

3. Right IF-rings. $R$ is said to be a right IF-ring if every injective right $\boldsymbol{R}$-module is flat. QF-rings, regular rings are trivial examples of right and left IF-rings. Moreover, $\operatorname{Hom}_{R}\left(F_{R}, F_{R}\right)$, for $F_{R}$ a nonfinitely generated free right $R$-module and $R$ a QF-ring, is a left IF-ring, by Sandomierski [11] and Lenzing [9].

Proposition 3.1. A right IF-domain is a field. 
Proof. In a domain, flat modules are torsion free. Thus every injective, being flat, is torsion free. Hence every module is nonsingular. For an essential right ideal $I, R / I$ is nonsingular, so $I=R$, implying that $R$ is a semisimple ring, hence a field, completing the proof. (Also follows from 3.3.)

$A$ ring $R$ is defined to be $Q F$ if every right and left ideal is an annulet and if it is right or left noetherian or artinian (and then it is both). As characterized by Faith and Walker [5], every injective module is projective.

By Bass [2], $R$ is right perfect in case every right $R$-module has a projective cover (dual to injective hull) and characterized as a ring with descending chain condition on finitely generated left ideals.

THEOREM 3.2. A right IF-ring $R$ is $Q F$ iff $R$ is right perfect.

Proof. By Bass [2, Theorem P], every flat right $R$-module is projective. Thus every injective is projective, hence QF, by [5, Theorem 5.3].

$\Leftarrow$ Since QF is artinian, $R$ is right perfect. For every finitely presented left $R$-module and injective right $R$-module $M$, one has a canonical homomorphism [4, p. 120]

$$
\operatorname{Tor}_{1}(M, F)=\operatorname{Tor}_{1}(\operatorname{Hom}(R, M), F) \rightarrow \operatorname{Hom}\left(\operatorname{Ext}^{1}(F, R), M\right)
$$

which is an epimorphism, as is easily verified (cf. [12]).

THEOREM 3.3. A right IF-ring is left FP-injective.

Proof. By above remark, $\operatorname{Hom}\left(\operatorname{Ext}^{1}(F, R), M\right)=0$. Choosing $M$ to be an injective cogenerator, we get $\operatorname{Ext}^{1}(F, R)=0$, proving the result.

In order to obtain the above result under the hypothesis of left perfect, we are forced to assume that a ring $R$ is right pseudocoherent in the sense that $r(S)$ is a finitely generated right ideal, for each finite subset $S$ of $R$.

THEOREM 3.4. A right IF, right pseudocoherent ring is $Q F$ iff $R$ is left perfect.

Proof. Follows by 3.3 and Björk [3, Theorem 4.3].

THEOREM 3.5. A right IF-ring is regular iff $w g l ~ R \leqq 1$.

Proof. Since every finitely generated right $R$-module is embeddable in a flat module, the result follows.

LemMA 3.6. $R$ is right IF-ring iff every right $R$-module is embeddable in a flat module.

THEOREM 3.7. A semiprime right Goldie ring $R$ is semisimple artinian iff $R$ is right IF-ring.

Proof. $\Rightarrow$ Obvious. 
$\Leftarrow$ By 3.3, $R$ is left FP-injective. Thus $R$ is left $R$-divisible (the proof of every injective left $R$-module is divisible also works for left FP-injective modules). Hence every regular element has a right inverse and thus is invertible. Since in a semiprime Goldie ring every essential right ideal has a regular element, (Goldie [7, Theorem 3.9]), which is invertible in our case, then $R$ has no essential right ideals. Hence $R$ is semisimple artinian.

PROPOSITION 3.8. Let $R$ be a cogenerator in the category of right $R$ modules. Then $R$ is right IF iff $R$ is left coherent.

Proof. $R$ is a cogenerator implies that every right $R$-module is torsionless. Since $R$ is $\Pi$-flat every module is embeddable in a flat module. Thus $R$ is right IF, by 3.6 .

The next corollary generalizes Faith's characterization of a QF-ring as a right or left self-injective ring with ascending chain condition on left annulets (cf. [6]).

COROLlaRY 3.9. A right IF-ring $R$ is $Q F$ iff $R$ has ascending chain condition on left annihilators.

Proof. Follows by 3.3 and [3, Theorem 4.1].

The same proof of Stenström [12, Proposition 4.2] yields:

THEOREM 3.10. In a left coherent ring, $R$ is right IF iff $R$ is left FPinjective.

4. Flat duals. Let (*) be a property that the dual of every right $R$ module is left $R$-flat. The next lemma follows immediately.

LEMMA 4.1. If $R$ is a ring with property (*), then $R$ is right coherent.

Proposition 4.2. Let $R$ have $\left({ }^{*}\right)$. Then the following are equivalent:

(1) $R$ is right FP-injective.

(2) $R$ is left FP-injective and left coherent.

(3) $R$ is regular.

Proof. $\quad(1) \Rightarrow(3)$. By 4.1 and 2.4 , every finitely presented left $R$ module $M$ is reflexive. $\left({ }^{*}\right) \Rightarrow M^{* *}$ is left $R$-flat. Thus $M$ is flat. Since every module is a direct limit of finitely presented modules, every module is flat.

(3) $\Rightarrow$ (1) and (2). Trivial.

(2) $\Rightarrow(3)$ follows symmetry to $(1) \Rightarrow(3)$.

THEOREM 4.3. For a right FP-injective ring $R$, the following are equivalent:

(a) $R$ has the property $\left({ }^{*}\right)$.

(b) $R$ is regular.

(c) $R$ is right coherent and wgl $R<\infty$.

(d) $R$ is right semihereditary. 
Proof. (a) $\Leftrightarrow$ (b) by 4.2 .

(b) $\Leftrightarrow$ (c) by Stenström [12, Proposition 3.6].

(b) $\Leftrightarrow$ (d) by Gupta [8, Theorem 3.4].

In conclusion, I would like to call attention to the following open problem. We remark that we have completely characterized left coherent right IF-rings ideal-theoretically via Theorem 3.10 , inasmuch as both left FP-injectivity and left coherency are so characterizable. However, we do not know whether a right IF-ring is left coherent, even for commutative rings.

\section{REFERENCES}

1. M. Auslander, Coherent functors, Proc. Conf. Categorical Algebra (La Jolla, Calif., 1965), Springer, New York, 1966, pp. 189-231. MR 35 \#2945.

2. H. Bass, Finitistic dimension and a homological generalization of semi-primary rings, Trans. Amer. Math. Soc. 95 (1960), 466-488. MR 28 \#1212.

3. J. E. Björk, Rings satisfying certain chain conditions, J. Reine Angew. Math. 245 (1970), 63-73. MR 43 \#3295.

4. H. Cartan and S. Eilenberg, Homological algebra, Princeton Univ. Press, Princeton, N.J., 1956. MR 17, 1040.

5. C. Faith and E. Walker, Direct sum representations of injective modules, J. Algebra 5 (1967), 203-221. MR 34 \#7575.

6. C. Faith, Rings with ascending condition on annihilators, Nagoya Math. J. 27 (1966), 179-191. MR 33 \#1328.

7. A. W. Goldie, Semi-prime rings with maximum condition, Proc. London Math. Soc. (3) 10 (1960), 201-220. MR 22 \#2627.

8. R. N. Gupta, On f-injective modules and semi-hereditary rings, Proc. Nat. Inst. Sci. India Part A 35 (1969), 323-328. MR 40 \#5658.

9. H. Lenzing, Über kohärente Ringe, Math. Z. 114 (1970), 201-212. MR 41 \#5410.

10. J. Lipman, Free derivation modules on algebraic varieties, Amer. J. Math. 87 (1965), 874-898. MR 32 \#4130.

11: F. L. Sandomierski, Some examples of right self-injective rings which are not left self-injective, Proc. Amer. Math. Soc. 26 (1970), 244-245. MR 42 \#324.

12. B. Stenström, Coherent rings and FP-injective modules, J. London Math. Soc. (2) 2 (1970), 323-329. MR 41 \#3533.

13. Y. Utumi, On quotient rings, Osaka Math. J. 8 (1956), 1-18. MR 18, 7.

Department of Mathematics, Rutgers University, New Brunswick, New JERSEY 08903

Current address: Department of Mathematics, University of Southern Illinois, Carbondale, Illinois 62901 